\title{
AVALIAÇÃO DO DESEMPENHO PSICOMOTOR DE CRIANÇAS HEMIPARÉTICAS ESPÁSTICAS ANTES E APÓS INTERVENÇÃO
}

Fabiana Araújo Silva, Mileide Cristina Stoco de Oliveira, Augusto Cesinando de Carvalho, Lúcia Martins Barbatto, Nayara Galvão Oliveira, Tânia Cristina Bofi

Universidade Estadual Paulista Júlio de Mesquita Filho - FCT/UNESP. Departamento de Fisioterapia, Presidente Prudente-SP.E-mail: fabyana_as@hotmail.com

\section{RESUMO}

A paralisia cerebral pode acarretar deficiências neuromotores e limitações no desempenho das áreas neuropsicomotoras. Podendo interferir na interação da criança com o meio, influenciando na a aquisição e no desempenho dos marcos motores básicos e atividades de vida diária. $O$ estudo objetivou avaliar o desempenho motor de crianças com diagnóstico de Paralisia Cerebral do tipo hemiparéticas espásticas antes e após a intervenção da psicomotricidade. Participaram do estudo oito crianças do sexo masculino com média idade cronológica de $90 \pm 18$ meses. Os instrumentos utilizados para avaliação foram a Escala de Desenvolvimento Motor e o Sistema de Classificação da Função Motora Grossa. Os participantes avaliados foram classificados como nível I de acordo com a GMFCS. A média da idade motora geral antes da intervenção era $50 \pm 16$ meses e após a intervenção $64 \pm 16$ meses. Os resultados do estudo permitiram analisar os déficits psicomotores dessa população. Evidenciou a viabilidade da avaliação para o planejamento dos programas de intervenção e a importância da psicomotricidade como proposta terapêutica para a população estudada.

Palavras-chave: Paralisia Cerebral, Desempenho Psicomotor, Modalidades da Fisioterapia.

\section{ASSESSMENT OF PSYCHOMOTOR PERFORMANCE SPASTIC HEMIPARETIC CHILDREN BEFORE AND AFTER INTERVENTION}

\begin{abstract}
Cerebral palsy can cause neuromotor impairments and limitations in the performance of neurological and psychomotor areas. May interfere with the child's interaction with the environment, influencing the acquisition and performance of motor milestones and basic activities of daily living. The study aimed to evaluate the motor performance of children diagnosed with cerebral palsy of spastic hemiparesis type before and after the intervention psychomotor. The study included eight male children with chronological age of $90 \pm 18$ months. The instruments were used to assess the scale of Motor Development and Classification System Gross Motor Function. The participants were evaluated classified as level I according to GMFCS. The overall motor age before the intervention was $50 \pm 16$ months after the intervention and $64 \pm 16$ months. The study results helped analyze the psychomotor deficits of this population. Demonstrated the feasibility of assessment for planning of intervention programs and the importance of psychomotor therapy as proposed for the population studied.
\end{abstract}

Keywords: Cerebral Palsy, Psychomotor Performance, Physical Therapy Modalities. 


\section{INTRODUÇÃO}

A paralisia cerebral (PC) é um distúrbio da postura e do movimento, resultante de encefalopatia não progressiva, com localização única ou múltipla no cérebro imaturo $^{1,2,3,4}$. A PC acomete o indivíduo de diferentes formas, dependendo da área do sistema nervoso afetada ${ }^{5}$. Pode determinar alterações neuromusculares, como variações de tono muscular, persistência de reflexos primitivos, rigidez, espasticidade, entre outros $^{4}$. Tais alterações geralmente se manifestam com padrões específicos de postura e de movimentos que podem comprometer o desempenho funcional dessas crianças $^{4,5}$. Consequentemente, a PC pode interferir de forma importante na interação da criança em contextos relevantes, influenciando na a aquisição e no desempenho dos marcos motores básicos e atividades de vida diária (AVD).

Além das deficiências neuromotoras, a paralisia cerebral pode também resultar limitações no desempenho das áreas neuropsicomotoras: motricidade fina, motricidade global, equilíbrio, esquema corporal, organização temporal e organização espacial.

Devido a todas essas alterações em crianças com PC, algumas AVDs podem ser prejudicas. Informações sobre o desempenho de atividades funcionais são extremamente relevantes, uma vez que as dificuldades no desempenho são, geralmente, a queixa principal de crianças, pais e familiares. Portanto, a promoção do desempenho de atividades e tarefas funcionais pode ser definida como objetivo a ser alcançado pelas terapêuticas empregadas ${ }^{6,7}$.

Mesmo que todas as alterações causadas por sequela da PC sejam citadas pela literatura, há ainda uma carência de definições e explicações, no sentido de identificar e relacionar como são os comprometimentos que esta patologia exerce sobre as aquisições da estabilidade, bem como nas AVDs. Esse estudo tem como objetivo avaliar o desenvolvimento motor de crianças com diagnóstico de PC do tipo hemiparéticas espásticas antes e após a intervenção da psicomotricidade.

\section{MÉTODOS}

Participaram do estudo 8 crianças com média idade cronológica de $90 \pm 18$ meses do sexo masculino, atendidas no Centro de Estudos e Atendimentos em Fisioterapia e Reabilitação (CEAFiR) da Faculdade de Ciências e Tecnologia FCT/UNESP. A participação na pesquisa foi autorizada pela assinatura do Termo de Consentimento Livre e Esclarecido aprovado pelo Comitê de Ética em Pesquisa pelos pais ou responsáveis. Esse projeto está protocolado (CAAE: 25877314.4.0000.5402) no Comitê de Ética em Pesquisa da 
FCT/UNESP. Apresenta como critérios de inclusão: crianças de 2 a 11 anos de idade com diagnóstico de PC e cujos pais autorizassem a realização da pesquisa. E os de exclusão foram: crianças com disfunções sensoriais, deficiência auditiva e ótica e alto grau de deficiência mental. Para classificar a gravidade do comprometimento neuromotor das crianças foi utilizado o Sistema de Classificação da Função Motora Grossa $(\mathrm{GMFCS})^{8}$, proposto por Palisano et al. Por meio desse instrumento, as crianças são agrupadas de acordo com os seguintes níveis de severidade: I e II que correspondem àquelas crianças com menor limitação da função e que conseguem deambular sem restrições; III corresponde àquelas crianças que necessitam de auxílio; IV e $\mathrm{V}$ abrange as crianças que se utilizam de tecnologia assistiva para se locomover.

Para avaliação do desenvolvimento das áreas neuropsicomotoras foi aplicado a Escala de Desenvolvimento Motor $(E D M)^{9}$, proposta por Rosa Neto (2002). A EDM compreende uma bateria de testes muitos diversificados e de dificuldade graduada, conduzindo a uma exploração minuciosa de diferentes setores do desenvolvimento. Avalia crianças dos 2 a 11 nas seguintes áreas: motricidade fina, motricidade global, equilíbrio, esquema corporal, organização espacial e temporal.
A distribuição dos dados foi dada por meio do teste Shapiro-Wilk. A comparação dos dados foi feita por meio teste $t$ de Student para dados pareados e para os dados não pareados foi utilizado One-way ANOVA seguido do teste de Tukey. O nível de significância considerado foi de $5 \%$.

\section{RESULTADOS}

Os participantes avaliados foram classificados como nível I de acordo com a GMFCS.

Os resultados da EDM demonstraram que as crianças participantes obtiveram média de idade motora geral (IMG) de 50 \pm 16 meses antes da intervenção e $64 \pm 16$ meses após a intervenção, com diferença estatisticamente significante $(p=0,001)$.

Os resultados encontrados em cada área motora avaliada na EDM (motricidade fina - IM1, motricidade global - IM2, equilíbrio - IM3, esquema corporal - IM4, organização temporal - IM5 e organização espacial IM6) e sua respectiva comparação entre os períodos de pré e pós intervenção da psicomotricidade estão representados na Tabela 1. Houve uma diferença significante na comparação entre o período de antes e após intervenção da psicomotricidade em todas as áreas avaliadas $(p<0,005)$. 
Tabela 1. Idades de cada área motora encontradas nos participantes avaliados apresentadas em meses

\begin{tabular}{ccccccc}
\hline Período & IM1 & IM2 & IM3 & IM4 & IM5 & IM6 \\
\hline $\begin{array}{c}\text { Antes } \\
\text { intervenção } \\
\begin{array}{c}\text { Após } \\
\text { intervenção }\end{array}\end{array}$ & $54 \pm 23^{*}$ & $51,43 \pm 16^{*}$ & $45,43 \pm 24^{*}$ & $49 \pm 13^{*}$ & $61 \pm 23^{*}$ & $45 \pm 15^{*}$ \\
\hline
\end{tabular}

* Diferença estatisticamente significante $(p<0,005)$

Os resultados da EDM referentes ao quociente motor (QM) de cada área motora avaliada EDM (motricidade fina - QM1, motricidade global - QM2, equilíbrio - QM3, esquema corporal - QM4, organização temporal - QM5 e organização espacial QM6) nos períodos pré e pós-intervenção da psicomotricidade estão apresentados na
Tabela 2. Houve uma diferença significante no quociente motor geral $50 \pm 16$ antes da intervenção e $71 \pm 12$ após a intervenção $(p<0,005)$. Os QM de todas as áreas motoras também obtiveram diferenças significantes no período de após intervenção comparado ao período de antes intervenção $(p<0,005)$.

Tabela 2. Média do Quociente motor de cada área avaliada antes e após intervenção.

\begin{tabular}{ccccccc}
\hline Período & QM1 & QM2 & QM3 & QM4 & QM5 & QM6 \\
\hline $\begin{array}{c}\text { Antes } \\
\text { intervenção } \\
\begin{array}{c}\text { Após } \\
\text { intervenção }\end{array}\end{array}$ & $66 \pm 28^{*}$ & $74 \pm 28$ & $56 \pm 27^{*}$ & $62 \pm 14^{*}$ & $74 \pm 21^{*}$ & $55 \pm 15^{*}$ \\
\hline
\end{tabular}

* Diferença estatisticamente significante $(p<0,005)$

\section{DISCUSSÃO}

A PC pode interferir de forma importante na interação da criança em contextos relevantes, influenciando na aquisição e no desempenho dos marcos do desenvolvimento motor básico e das AVDs, assim como nas habilidades neuromotoras.

$\mathrm{Na}$ análise do desenvolvimento motor da criança, em cada tarefa, pode-se observar um déficit motor, principalmente nas áreas de motricidade fina e equilíbrio corporal, as quais apresentaram um QM inferior à idade cronológica, resultado classificado pela EDM. Esse resultado corrobora aos encontrados na literatura $^{10}$, que em suas investigações com crianças com sequelas de PC, encontrou déficits significativos no desenvolvimento motor nas áreas citadas. 
No atual estudo as áreas de esquema corporal e organização espacial, encontraram-se QM considerados abaixo da idade cronológica das crianças avaliadas, no entanto, esse atraso foi menor quando comparado às áreas de motricidade fina e equilíbrio corporal. A área que apresentou menor déficit foi à organização temporal, com exceção de um participante. Percebendo-se assim, que este apresentou noção de tempo e ritmo quase no padrão de normalidade.

O esquema corporal é a consciência do corpo como elo de comunicação consigo e com o meio ${ }^{11}$ e indivíduos com diagnóstico de paralisia cerebral pode ter dificuldades de reconhecer as possibilidades de ação do próprio corpo. Nas crianças avaliadas apresentou-se abaixo das IC. Um adequado desenvolvimento desse esquema pressupõe uma evolução da motricidade pertinente ao crescimento e desenvolvimento do indivíduo, das percepções espaciais e temporais e da afetividade. A consciência adequada do corpo envolve a imagem e o conceito corporal. Essa consciência pode ser desenvolvida ao realizar atividades que favoreçam o controle e o conhecimento do corpo a utilizar seus seguimentos.

A maioria das crianças apresentou déficits motores nas áreas que foram avaliadas pela EDM, principalmente a área do equilíbrio. Há inúmeros fatores neurais que contribuem com as dificuldades de manutenção do equilíbrio na criança com hemiparesia espástica por paralisia cerebral, incluindo espasmo muscular, ou reflexos de extensão hiperativos com uma ativação aumentada dos músculos responsáveis pela postura $^{12,13}$. As alterações musculo esqueléticas com o déficit de flexibilidade e encurtamentos corroboram para o aumento de problemas da estabilidade.

Em alguns estudos que compararam o desenvolvimento do equilíbrio de crianças saudáveis e crianças com hemiparesia espástica, buscou-se determinar se a característica da postura contribui para uma resposta de padrões atípicos dos músculos de crianças com paralisia cerebral ${ }^{14,15}$. Os resultados indicam que para a simulação da postura da paralisia cerebral, em crianças saudáveis, ocorre também a ativação dos músculos antagonistas, assim como normalmente efetuado por crianças com hemiparesia espástica, o que prejudica as reações posturais deste grupo, com destaque para o equilíbrio corporal. Além da excessiva ativação muscular antagônica devido a fraqueza dos músculos agonistas, crianças com paralisia cerebral apresentam excessivos movimentos voluntários durante a locomoção.

A dificuldade da manutenção do equilíbrio de crianças com paralisia cerebral está relacionada principalmente aos déficits 
do sistema nervoso central e mudanças mecânicas do alinhamento do corpo. A ativação muscular pode ser citada também como um dos fatores intervenientes para a correta estabilidade corporal, bem como, as dificuldades de se manter determinada postura e/ou posicionamento dos segmentos corporais e mobilidade articular ${ }^{13,14}$.

\section{CONCLUSÃO}

A avaliação do desenvolvimento psicomotor de crianças com hemiparesia espásticas é de total importância para detecção de dificuldades nas áreas neuropsicomotoras como a motricidade fina, motricidade global, equilíbrio, esquema corporal, organização espacial e organização temporal. Ambas as áreas atuam na realização das AVDs, percepção da criança no tempo e espaço, além do desenvolvimento de habilidades cognitivas.

O resultado do desenvolvimento psicomotor das crianças avaliadas sugere uma importante relação entre as dificuldades motoras e as alterações do aspecto corporal atribuídos à PC. Apesar do tamanho da amostra ser reduzido, o estudo evidenciou a viabilidade dos resultados encontrados na avaliação para o planejamento dos programas de intervenção e a importância e eficiência da psicomotricidade como proposta terapêutica para a população estudada.

\section{REFERÊNCIAS}

1. Howle JMW. Cerebral palsy. In: Campbell SM, editor. Decision making in pediatric neurologic physical therapy. Nova York: Churchill Livingstone; 1999; 23-83.

2. Wright MJ, Olney SJ. Cerebral palsy. In: Campbell SK. Physical therapy for children. 2nd. Phyladelphia: WB Saunders company; 1995; 489-523.

3. Wilson JM. Cerebral palsy. In: Campbell SK. Clinics in physical therapy pediatric neurologic physical therapy. 2nd. New York: Churchill Livingstone; 1991; 30160.

4. Souza ACL. Terapia ocupacional em paralisia cerebral espástica. In: Sousa AMC, Ferraretto I. Paralisia cerebral: aspectos práticos. São Paulo: Mennon; 1998. p. 23142.

5. Bax MCO. Terminology and classification of cerebral palsy. Dev Med Child Neurol; $1964 ; \quad 6:$ 295-7. http://dx.doi.org/10.1111/j.1469-

8749.1964.tb10791.x

6. Dzienkowski RC, Smith KK, Dillow KA, Yucha CB. Cerebral palsy: a comprehensive review. Nurse practitioner 1996; 21: 45-61. http://dx.doi.org/10.1097/00006205199602000-00004

7. Mackie PC, Jessen EC, Jarvis SN. The lifestyle assessment questionnaire: an instrument to measure the impact of disability on the lives of children whit cerebral palsy and their families. Child Care Health Dev 1998; 24: 473-86. http://dx.doi.org/10.1046/j.13652214.1998.00083.x

8. Palisano $\mathrm{R}$, Rosenbaum $\mathrm{P}$, Walter $\mathrm{S}$, Russell D, Wood E, Galuppi B. Development and reliability of a system to classify gross motor function in children with cerebral palsy. Dev Med Child Neurol. 1997; 
39(4):214-23.

http://dx.doi.org/10.1111/j.1469-

8749.1997.tb07414.x

9. Rosa NF, Manual de Avaliação Motora. Porto Alegre: Artmed, 2002.

10. Ferreira MEC. Desenvolvimento perceptivo motor de crianças com Síndrome de Down e paralisia cerebral. Revista da Sobama. 1997; 2(2):17-22.

11. Fonseca V. "Desenvolvimento psicomotor e aprendizagem": Artmed. Gaio, R. \& Porto, E. Educação Física e Pedagogia do Movimento: possibilidades do corpo em diálogo com as diferenças. 2008. Campinas: De Marco; 2006. Educação Física: cultura e sociedade.

12. Blanche El. Fazer junto com - não fazer para: a recreação e as crianças portadoras de paralisia cerebral. In: Parham LD, Fazio LS. A recreação na Terapia Ocupacional pediátrica. São Paulo: Santos editora e livraria; 2000; 202-18.

13. Assaiante C. Development of locomotor balance control in healthy children. Neurosci and Biobehav Rev, Fatetteville. 1998; 2 (4): 527-32. http://dx.doi.org/10.1016/S01497634(97)00040-7

14. Mancini M.C, Alves, ACM. Schaper, C., Figueiredo EM., Sampaio RF, Coelho ZA C, Tirado MGA. Gravidade da paralisia cerebral e desempenho funcional. Rev. bras. fisioter. 2004; 8 (3): 253-60.

15. Woollacott $\mathrm{MH}$, Burtner $\mathrm{P}$, Jensen J, Jasiewics J, Roncesvalles $\mathrm{N}$, Sveistrup $\mathrm{H}$. Development of postural responses during standing in healthy children and children with spastic diplegia. Neurosci and Biobehav Rev, Fayetteville. 1998; 22 (4):583-9. http://dx.doi.org/10.1016/S0149-

7634(97)00048-1
Recebido para publicação em 19/08/2014 Revisado em 15/09/2014

Aceito em 17/09/2014 